\title{
Total resection of presacral giant schwannoma via minimally invasive dorsal approach: illustrative case
}

\author{
Guenther C. Feigl, MD, PhD, ${ }^{1,2,3}$ Domagoj Jugovic, MD, PhD, ${ }^{3,5}$ Daniel Staribacher, MD, ${ }^{3}$ Rolf Buslei, MD, ${ }^{4}$ and Dzmitry Kuzmin, $\mathrm{MD}^{3}$ \\ ${ }^{1}$ Department of Neurosurgery, University Hospital Tuebingen, Tuebingen, Germany; ${ }^{2}$ Department of Neurological Surgery, Houston Methodist Hospital, Houston, Texas; \\ Departments of ${ }^{3}$ Neurosurgery and ${ }^{4}$ Pathology and Neuropathology, General Hospital Bamberg, Bamberg, Germany; and ${ }^{5}$ Faculty of Medicine, University of Ljubljana, \\ Ljubljana, Slovenia
}

BACKGROUND Giant presacral schwannomas are extremely rare in neurosurgery. There are various approaches to the surgical treatment of symptomatic giant presacral schwannomas. The least traumatic is the one-stage surgery with a dorsal approach.

OBSERVATIONS The authors describe a case of a 52-year-old male with pain in the sacral region and partial urinary dysfunction. A total tumor resection through a minimally invasive dorsal approach was performed, and anatomical and functional preservation of all sacral nerves with no postoperative complications was achieved.

LESSONS The authors have shown the possibility of total tumor resection with a minimally invasive dorsal approach without the development of intraand postoperative complications. Operative corridors that have been created by a tumor can be used and expanded for a minimally invasive dorsal approach to facilitate resection and minimize tissue disruption.

https://thejns.org/doi/abs/10.3171/CASE21319

KEYWORDS presacral giant schwannoma; minimally invasive approach; dorsal approach

Schwannomas are tumors originating from Schwann cells of the nerve sheaths. Most commonly, schwannomas are slow-growing benign tumors that do not infiltrate the surrounding organs and tissues but cause their compression. ${ }^{1,2}$ Approximately $60 \%$ of all schwannomas are vestibular schwannomas. ${ }^{3}$ Sacral spine schwannomas are quite rare and usually asymptomatic. They become symptomatic over time when they reach a significant size and fill the lesser pelvis, compressing neighboring organs and neurovascular structures. ${ }^{4}$ Symptoms such as sacral pain, disorders of urination and defecation, and neurological symptoms in the lower extremities may occur.

Giant presacral schwannomas are extremely rare. Due to this fact, no common opinion about the surgical treatment approach exists. By analysis of the data from current literature, it was possible to establish that the ventral approach is preferable because it allows total resection to be achieved. ${ }^{5}$ However, the disadvantages of this approach are the risks of complications such as damage to vessels and pelvic organs.
The anterior approach is performed most commonly through a long skin incision and transection of abdominal muscles, which is required to achieve sufficient exposure of the schwannoma. This then results in a longer period of postoperative recovery for the patient. A dorsal approach can help to avoid such prolonged postoperative recovery periods. However, its main disadvantage is the limited surgical area. ${ }^{6}$ Therefore, surgeons often resort to multisegmental laminectomy and even partial resection of the sacrum with subsequent stabilization. ${ }^{7,8}$ Depending on the position of the tumor in the lower pelvis, it is not always possible to achieve total one-stage tumor resection through the dorsal approach. ${ }^{9}$ We present a clinical case report of giant presacral schwannoma and total resection with a minimally invasive dorsal approach.

\section{Illustrative Case}

For many years, a 52-year-old patient had pain in the sacrum and gluteal region on the right. In recent months, he also complained of urinary problems, namely urinary urgencies. Clinical 
examination revealed no neurological impairments. However, ultrasound examination of the lesser pelvis then showed a round, space-occupying lesion. Therefore, further imaging, including magnetic resonance imaging (MRI) and computed tomography (CT) angiography of the pelvis, was performed. The imaging revealed a presacral lesion that was $9.4 \times 9 \times 7.5 \mathrm{~cm}$ in size, hypointense on T1-weighted images (Fig. 1), and hyperintense on T2-weighted images with contact to the right S2 nerve root. A perforation of the sacrum on the right side was visible. CT angiography showed close contact with the iliac vessels but without infiltration.

A minimally invasive dorsal approach was chosen for surgical treatment. See Fig. 2 for 3-dimensional image reconstruction using the Surgical Theater visualization platform. It was possible to achieve a total resection of the tumor, which was confirmed by postoperative MRI scans (Fig. 3). The right S2 nerve root was anatomically and functionally preserved, which was confirmed with intraoperative direct stimulation. No neurological impairments were observed in the early postoperative period. The patient reported an improvement of his bladder function. The histopathological examination revealed a schwannoma (Fig. 4).

\section{Surgical Technique}

The surgery was carried out with the patient lying on his stomach (prone position). The third sacral vertebra was localized and identified by radiography. The skin incision was only approximately $3.5 \mathrm{~cm}$ long (Fig. 5). A special wound retractor called a Feigl retractor (Fehling) was used for the minimally invasive approach. A hemilaminectomy was performed, and the schwannoma, which compressed the right S2 root from the lateral side, was microsurgically resected, exposing the S2 root. The S2 root was visualized and identified using

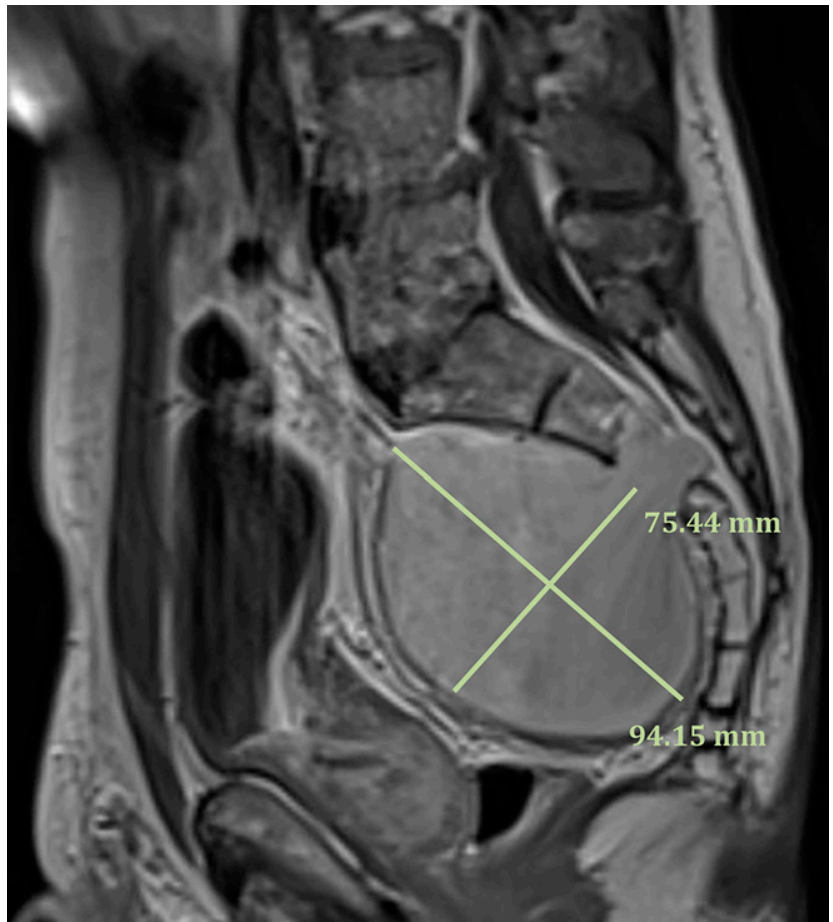

FIG. 1. Preoperative imaging. T1-weighted image with contrast medium in the sagittal section. The presacral tumor measured $9.4 \mathrm{x}$ $9.0 \times 7.5 \mathrm{~cm}$ in size, originating from the right $\mathrm{S} 2$ nerve root.

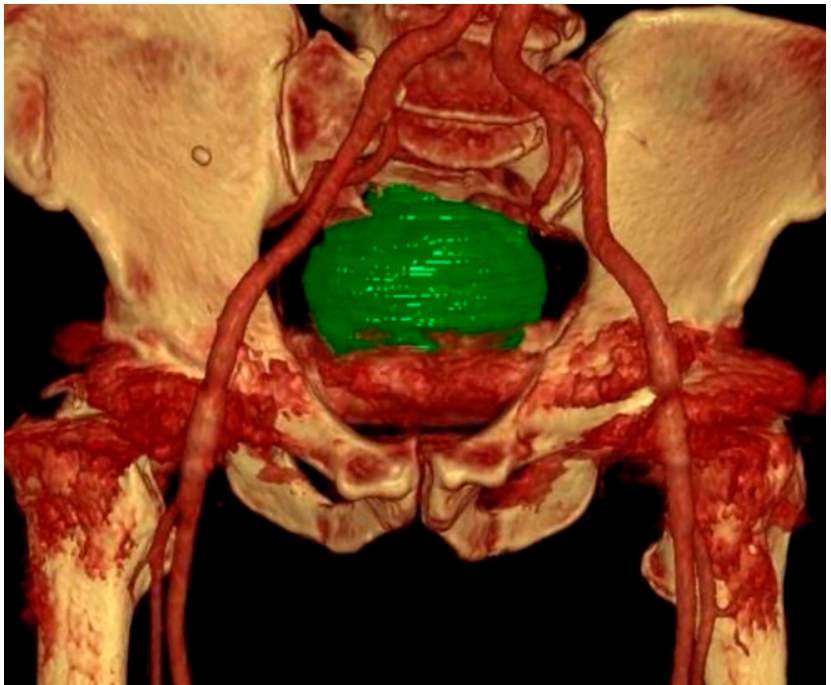

FIG. 2. 3-Dimensional image reconstruction obtained with the Surgical Theater system in the coronal plane.

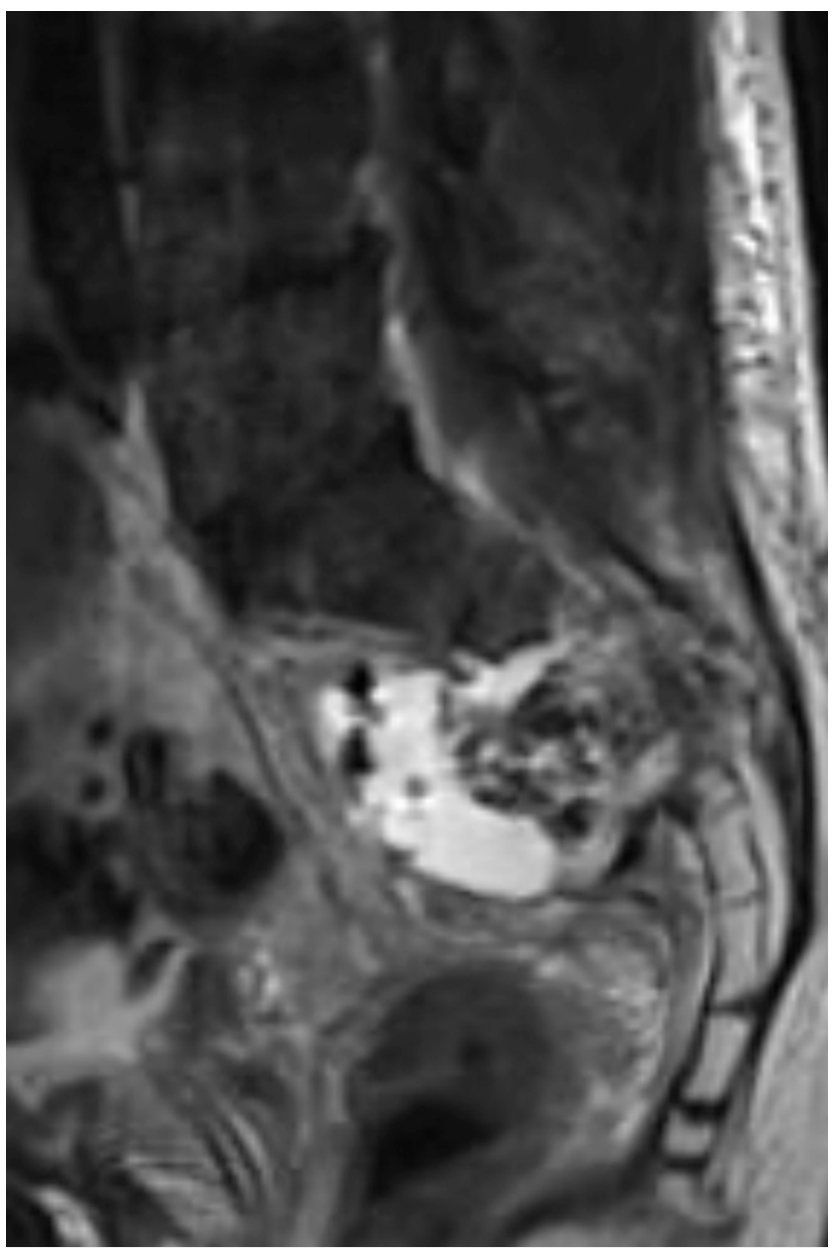

FIG. 3. Postoperative MRI of the lesser pelvis. T2-weighted image in the sagittal plane shows small seroma in the area of the removed tumor. 


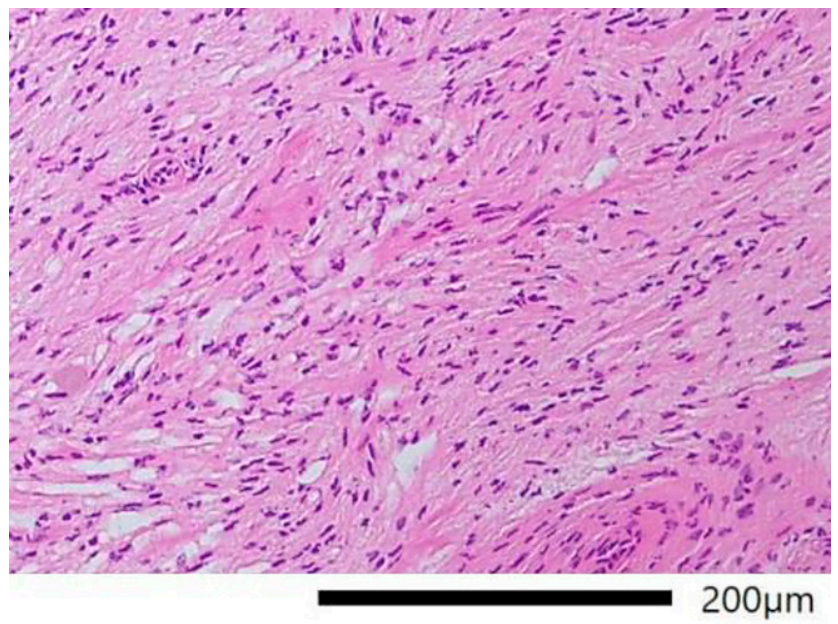

FIG. 4. Histology: schwannoma, WHO grade I.

direct nerve stimulation. After resection of the tumor parts that infiltrated the sacrum, the tumor was followed down into the retroperitoneal presacral space. The tumor had the typical tissue structure and yellowish color of a schwannoma. The tissue consistency of the tumor varied from necrotic to solid to soft. With the help of various microsurgical instruments and dissectors, the tumor was carefully peeled off the surrounding intraabdominal anatomical structures. After complete tumor removal, the resection area was examined using the QEVO endoscopic inspection tool, and it was confirmed that a gross total resection was achieved. The resection cavity, which shrank after the tumor was removed, was filled with TachoSil hemostatic substance and additionally covered with some fibrin glue. Then the wound was sutured layer by layer intracutaneously, and the skin was sealed with Dermabond (see Video 1).

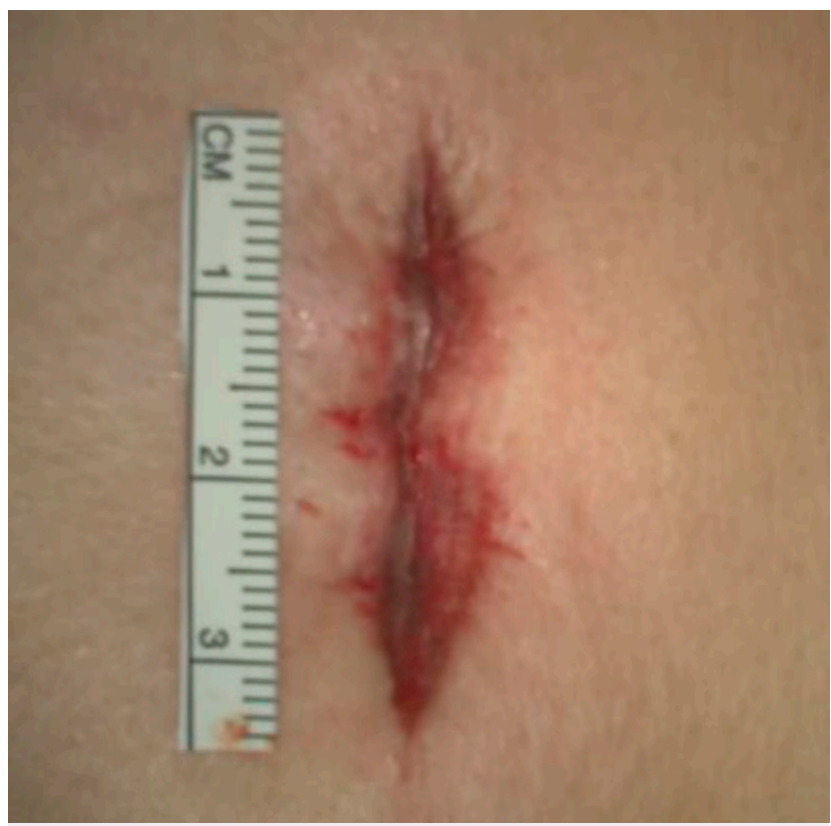

FIG. 5. The size of the operating wound is $3.5 \mathrm{~cm}$.
VIDEO 1. Clip showing total resection of presacral giant schwannoma via minimally invasive dorsal approach. (1) 3Dimensional image reconstruction using Surgical Theater. (2) One sees a presacral giant schwannoma and bone degradation from the tumor. Surgery: A hemilaminectomy is performed first. Then the S2 nerve root is stimulated directly. Then the tumor is dissected from the nerve root, and the tumor capsule is opened. Thereafter, the tumor is gradually removed. The opening is covered with TachoSil and fibrin glue. At the end of the operation, an S2 nerve root is again stimulated. Click here to view.

\section{Discussion}

The strategy of surgical treatment and the choice of a suitable approach mainly depend on the characteristics of the tumor: its size, localization in relation to the vessels and organs of the lesser pelvis, and its consistency. ${ }^{10,11}$ However, the surgical experience of the team of surgeons also plays a significant role. Taking into account that giant presacral schwannomas are extremely rare, there is not much experience with their surgical removal. The choice of a ventral approach with laparotomy is mainly conditioned by its simplicity for the surgeon because it is a classic approach in pelvic surgery. ${ }^{2,5,8,9,12}$ The dorsal approach primarily limits the surgical area. Therefore, surgeries are often accompanied by multisegmental laminotomies or resection of a part of a bone with subsequent stabilization., 6,9

Neurosurgery has recently been striving to reduce the surgical approach, which is directly related to a decrease in intraoperative risks and postoperative complications.

\section{Observations}

Our clinic specializes in focusing on minimally invasive methods, and extensive experience has been accumulated in the total removal of tumors of other localizations through minimally invasive approaches. As a result, we chose a minimally invasive approach to remove this giant presacral schwannoma. Using and expanding operative corridors that have been created by a tumor facilitated resection and minimized tissue disruption. Total removal was achieved while maintaining the function of the nerve root. The patient showed no new neurological impairments and was satisfied with the surgical treatment. The surgery can be considered completely curative.

However, this method has its constraints. The main issue is the detachment of the tumor from the sac, which makes it possible to exclude pelvic organ traumatization. This method cannot be used if the tumors are malignant. ${ }^{13}$

To our knowledge, this is the first report of a minimally invasive dorsal approach for total resection of a giant presacral schwannoma. This case opens up new horizons in the surgery of giant presacral schwannomas and can be used successfully in neurosurgical practice.

\section{Lessons}

Giant presacral schwannomas are extremely rare, and there are no unambiguous approaches to their surgical treatment. We have shown the possibility of total tumor resection with a minimally invasive dorsal approach without the development of intra- and postoperative complications. Operative corridors that have been created by a tumor can be used and expanded for a minimally invasive dorsal approach to facilitate resection and minimize tissue disruption. 


\section{References}

1. Choudry HA, Nikfarjam M, Liang JJ, et al. Diagnosis and management of retroperitoneal ancient schwannomas. World J Surg Oncol. 2009:7:12.

2. Kuriakose S, Vikram S, Salih S, Balasubramanian S, Mangalasseri Pareekutty N, Nayanar S. Unique surgical issues in the management of a giant retroperitoneal schwannoma and brief review of literature. Case Rep Med. 2014;2014:781347.

3. Propp JM, McCarthy BJ, Davis FG, Preston-Martin S. Descriptive epidemiology of vestibular schwannomas. Neuro Oncol. 2006;8(1):1-11.

4. Tish S, Habboub G, Lang M, et al. The epidemiology of spinal schwannoma in the United States between 2006 and 2014. J Neurosurg Spine. 2019;32(5):661-666.

5. Santiago C, Lucha PA. Atypical presentation of a retrorectal ancient schwannoma: a case report and review of the literature. Mil Med. 2008;173(8):814-816.

6. Zang J, Guo W, Yang Y, Wei R. Surgical treatment of giant benign sacral neurogenic tumors using the posterior-only approach. Clin Neurol Neurosurg. 2019;185:105483.

7. Emohare O, Stapleton M, Mendez A. A minimally invasive pericoccygeal approach to resection of a large presacral schwannoma: case report. J Neurosurg Spine. 2015;23(1):81-85.

8. Sun W, Ma XJ, Zhang F, Miao WL, Wang CR, Cai ZD. Surgical treatment of sacral neurogenic tumor: a 10-year experience with 64 cases. Orthop Surg. 2016;8(2):162-170.

9. Pongsthorn C, Ozawa H, Aizawa T, Kusakabe T, Nakamura T, Itoi E. Giant sacral schwannoma: a report of six cases. Ups J Med Sci. 2010;115(2):146-152.

10. Li Q, Gao C, Juzi JT, Hao X. Analysis of 82 cases of retroperitoneal schwannoma. ANZ J Surg. 2007;77(4):237-240.
11. Schindler OS, Dixon JH, Case P. Retroperitoneal giant schwannomas: report on two cases and review of the literature. $J$ Orthop Surg (Hong Kong). 2002;10(1):77-84.

12. Samarakoon L, Weerasekera A, Sanjeewa R, Kollure S. Giant presacral schwannoma presenting with constipation: a case report J Med Case Rep. 2012;6:285.

13. Lee BH, Hyun S-J, Park J-H, Kim K-J. Single stage posterior approach for total resection of presacral giant schwannoma: a technical case report. Korean J Spine. 2017;14(3):89-92.

\section{Disclosures}

The authors report no conflict of interest concerning the materials or methods used in this study or the findings specified in this paper.

\section{Author Contributions}

Conception and design: Feigl, Jugovic, Staribacher, Kuzmin. Acquisition of data: Feigl, Jugovic, Buslei, Kuzmin. Analysis and interpretation of data: Feigl, Jugovic, Kuzmin. Drafting the article: Jugovic, Kuzmin. Critically revising the article: Feigl, Jugovic, Staribacher. Reviewed submitted version of manuscript: Feigl, Jugovic, Staribacher. Approved the final version of the manuscript on behalf of all authors: Feigl. Administrative/technical/material support: Feigl, Buslei. Study supervision: Feigl.

\section{Supplemental Information \\ Video}

Video 1. https://vimeo.com/566609287.

\section{Correspondence}

Guenther C. Feigl: University Hospital Tuebingen, Tuebingen, Germany. guenther.feigl@sozialstiftung-bamberg.de. 